Article

\title{
Evolutionary Coalitional Game-Based Cooperative Localization in Vehicular Networks
}

\author{
Ting Yin ${ }^{1,2, * \mathbb{D}}$, Decai Zou ${ }^{1,3}$, Xiaochun $\mathrm{Lu}^{1,3}$ and Cheng $\mathrm{Bi}^{4}$ \\ 1 National Time Service Center, Chinese Academy of Sciences, Xi'an 710600, China; zdc@ntsc.ac.cn (D.Z.); \\ 1xc@ntsc.ac.cn (X.L.) \\ 2 School of Electronic, Electronial and Communication Engineering, University Chinese Academy of Sciences, \\ Beijing 100049, China \\ 3 School of Astronomy and Space Science, University Chinese Academy of Sciences, Beijing 100049, China \\ 4 School of Telecommunications Engineering, Xidian University, Xi'an 710071, China; bicheng_pro@126.com \\ * Correspondence: yinting@ntsc.ac.cn
}

Citation: Yin, T.; Zou, D.; Lu, X.; Bi, C. Evolutionary Coalitional

Game-Based Cooperative

Localization in Vehicular Networks. Electronics 2022, 11, 638. https:// doi.org/10.3390/electronics11040638

Academic Editor: Juan-Carlos Cano

Received: 27 January 2022

Accepted: 17 February 2022

Published: 18 February 2022

Publisher's Note: MDPI stays neutral with regard to jurisdictional claims in published maps and institutional affiliations.

Copyright: (c) 2022 by the authors. Licensee MDPI, Basel, Switzerland. This article is an open access article distributed under the terms and conditions of the Creative Commons Attribution (CC BY) license (https:// creativecommons.org/licenses/by/ $4.0 /)$.

\begin{abstract}
Cooperative localization under complex urban environments has become a solution able to replace Global Navigation Satellite System (GNSS) positioning. Due to the lack of an efficient cooperative localization scheme, traditional cooperative vehicle networks result in high computational complexity and heavy communication overhead. In this paper, we concentrate on the cooperative localization design of a vehicle. This paper proposes a cooperative localization method based on evolutionary coalitional game theory to implement vehicle location estimation with a lower communication cost. We select the neighboring vehicles to form a coalition based on the node's square position error bound and communication cost. The location is obtained via exchanging information between vehicles. It is evident from the simulations and results that the proposed method requires a low communication overhead while maintaining localization accuracy.
\end{abstract}

Keywords: cooperative localization; vehicle network; game theory; evolutionary coalitional game

\section{Introduction}

With the Intelligent Transportation System (ITS) developing rapidly, vehicle positioning technology has become a focus for autonomous driving applications [1,2]. In these applications, the vehicle is generally located with Global Navigation Satellite Systems (GNSS). However, GNSS signals are usually poor in urban canyons and overpasses due to high-rise building occlusion and multipath issues. Therefore, the demand for localization accuracy under dense and complex urban environments has become high.

There are many novel localization methods used in vehicle networks to deal with complex urban environments. Visual localization is a standard technology used to match and predict vehicles' positions via identifying and searching local features [3]. Similarly, radar technology is widely used in dual-functional radar communication (DFRC) systems. It is superior to reducing the signaling overhead and improving the angle estimation accuracy [4]. Since different positioning technologies are suitable for urban environments, multi-data fusion has become inevitable. The fusion of multiple types of localization information can effectively deal with complex urban environments [5,6].

With the development of the fifth generation of mobile communication (5G), 5G mmWave positioning has become a new localization scheme [7] that is generally categorized as a base station (BS) -based method and user-based method [8]. The BS-based method applies the Location Management Function to estimate users' positions. However, vehicles often fail to be located due to network congestion and high latency occurring in the dense urban environments and crowded situations in which many vehicles access the BS. Inversely, the user-based method is an active form of localization in which the user receives 
positioning signals and calculates their position. Therefore, the user-based method is more reliable than the BS-based method for vehicle networks.

Cooperative vehicle localization is an effective model for the user-based method [9-11]. In general, both non-Bayesian-based and Bayesian-based methods are considered in cooperative localization. In non-Bayesian models, widespread estimators include the least-squares (LS) method and the maximum likelihood (ML) method. For the Bayesian-based model, there are belief propagation and nonparametric belief propagation [12,13]. It is noticed that the Bayesian-based method performed well compared to the non-Bayesian method in terms of localization accuracy. The classical algorithm of the Bayesian-based method is the sum-product algorithm over a wireless network (SPAWN) [12], which estimates the position via the anchors' position information and the posterior probability of the nodes through message passing. In [14], the authors employ the idea of the posterior linearization filter in belief propagation with the sigma-point method. The posterior linearization belief propagation (PLBP) method performs well for the localization accuracy with nonlinear measurement. However, the cost of communication and the computational complexity increase under the scenarios with numerous nodes, and it cannot meet the requirements of these use cases.

A number of relevant problems have lately received considerable attention in cooperative localization. Game theory is successful in solving various limitations. In [15], the authors propose an improved game theory-based cooperative localization algorithm for multi-robot system localization. In [16], the authors propose a network formation game for cooperative localization to reduce the computational complexity and resultant network traffic. Similarly, the coalition game, a typical static game theory, is widely implemented for cooperative localization [17]. In addition to that, the most informative agents for exchanging information are determined with the opportunistic cooperative localization-based overlapping coalition formation (OCF) game $[18,19]$. However, the above game theories are static games that need to reach equilibrium with a heavy communication overhead every time the topology changes. They are unsuitable for networks with dynamic topology, such as vehicle networks. Therefore, we embed the dynamic game theory in cooperative localization and propose a framework for solving the problem of communication cost.

The main contribution of this paper is to address the problem of the high computation complexity and communication cost for vehicle networks in cooperative localization. In this paper, we formulate the cooperative vehicle localization problem as an evolutionary coalitional (EC) game. The vehicles autonomously decide whether to assist other vehicles based on their positioning performance and the current position relationship. The simulation results demonstrate that the proposed method performs better than the PLBP algorithm. It is noticed that the proposed method reduces the computation complexity and communication cost while maintaining the same accuracy.

\section{Materials and Methods}

\subsection{Network Model}

The crossroads congestion scenarios in the city are described in Figure 1. Due to density of high buildings, vehicles cannot receive satellite signals with a good geometric dilution precision. The scenario comprises M Roadside unit (RSUs) and N vehicles. In detail, the RSU is considered a small base station with precise location and clock information, and the vehicles are within the coverage of the RSU. Significantly, the vehicle must be time-synchronized with the RSU and randomly deployed with unknown positions.

In this scenario, 5G mmWave and multiple-input-multiple-output (MIMO) provide the position and orientation [20], respectively. Additionally, accurate location and synchronization information are contributed for the RSUs via vehicle-to-infrastructure (V2I) communication. Vehicle-to-vehicle (V2V) communication is employed for distance measurement and message transformation. The distances model measured with time-difference-of-arrival (TDOA) is given as

$$
z_{i j, t}=h_{\mathrm{TDOA}}\left(x_{i, t}, x_{j, t}\right)+\beta_{i, t}+\beta_{j, t},
$$


where $x_{i, t}$ and $\beta_{i, t}$ denote the $i$ vehicle's location and the normally distributed process noise with $N\left(0, \sum_{\beta_{i, t}}\right)$ at the time $t$, respectively. The $h_{\mathrm{TDOA}}\left(x_{i, t}, x_{j, t}\right)$ is a function of both $x_{i, t}$ and $x_{j, t}$.

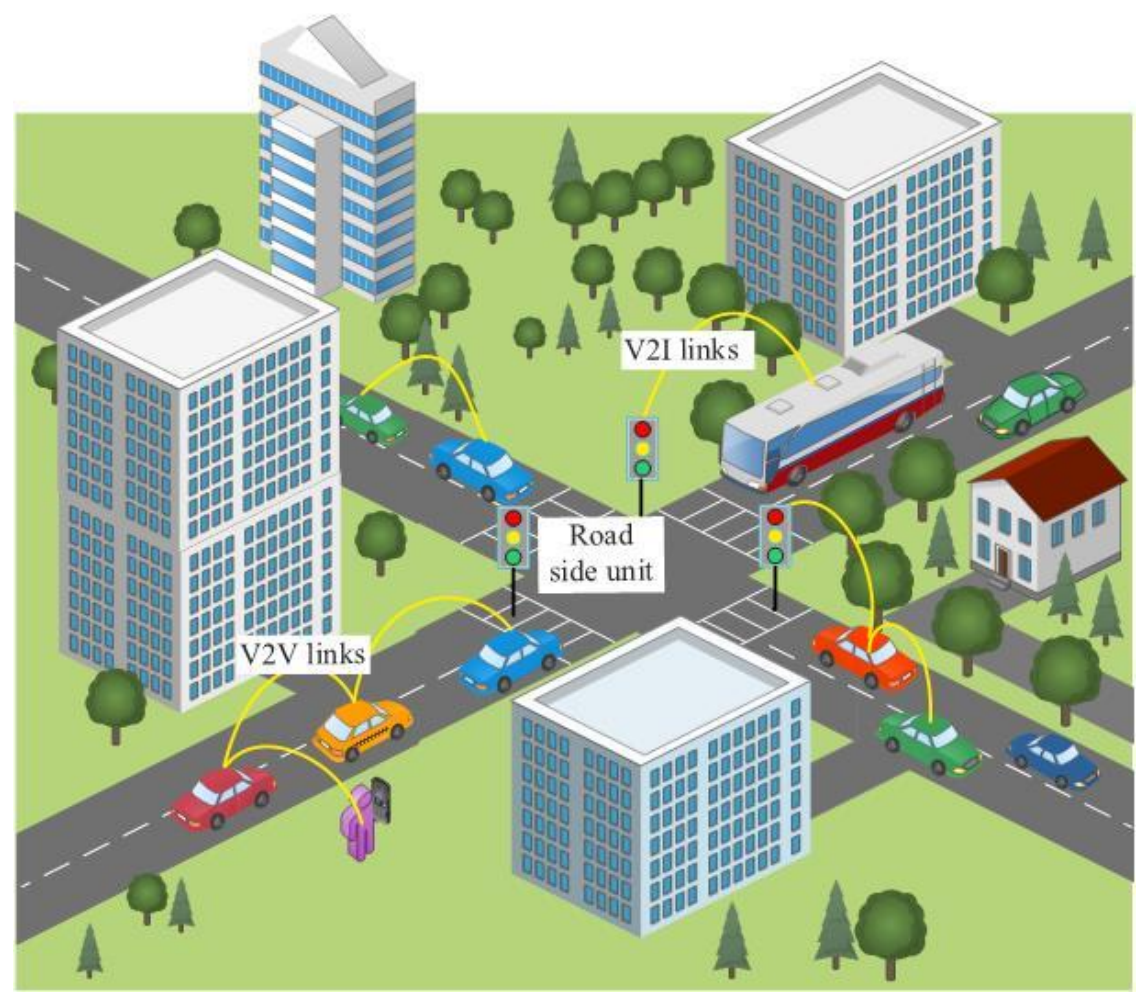

Figure 1. The system model of vehicular network.

The mobile state of the vehicle is a random walk model, and each vehicle has a fixed speed and direction. The following state $x_{i, t+1}$ has evolved from the current state $x_{i, t}$ according to

$$
x_{i, t+1}=x_{i, t}+\Delta t \cdot v_{i}+w_{i},
$$

where $\Delta t$ is the time step, $v_{i}$ is the velocity, and $w_{i}$ represents the process noise.

\subsection{Belief Propagation Algorithm for Cooperative Localization}

The cooperative localization algorithm based on the belief propagation (BP) algorithm is widely recognized with a high accuracy and suitable for static and dynamic network scenarios.

To be specific, the BP algorithm is based on factor groups and Gaussian message propagation, and is able to estimate the locations via exchanging the vehicle's statistical information with a Gaussian distribution $[12,14]$. In the beginning, the vehicle broadcasts discovery information and measures distances among the neighboring vehicles and RSUs. According to the vehicle's $i$ prior information, the message $\mu$ from factor $f_{i, t}$ to state $x_{i, t}$ at the $l$ th iteration in time $t$ is given in

$$
\mu_{f_{i, t} \rightarrow x_{i, t}}^{(l)}\left(x_{i, t}\right)=\int p\left(x_{i, t} \mid x_{i, t-1}\right) b^{(l-1)}\left(x_{i, t-1}\right) d x_{i, t-1} .
$$

The vehicle calculates the incoming message when receiving the messages from neighbors, and is given as:

$$
\mu_{f_{i j, t} \rightarrow x_{i, t}}^{(l)}\left(x_{i, t}\right)=\int p\left(z_{i j, t} \mid x_{i, t}, x_{j, t}\right) \prod_{j \in S_{i}} \mu_{x_{i, t} \rightarrow f_{i j, t}}^{(l-1)}\left(x_{i, t}\right) d x_{i, t} .
$$


The outgoing message is defined as:

$$
\mu_{x_{i, t} \rightarrow f_{i j, t}}^{(l)}\left(x_{i, t}\right)=\mu_{f_{i, t} \rightarrow x_{i, t}} \prod_{j \in S_{i} / j} \mu_{f_{i j, t} \rightarrow x_{i, t}}^{(l)}\left(x_{i, t}\right)
$$

Moreover, the posterior distribution $p(x \mid z)$ is denoted:

$$
b^{(l)}\left(x_{i, t}\right) \propto \mu_{f_{i, t} \rightarrow x_{i, t}}\left(x_{i, t}\right) \prod_{j \in S_{i}} \mu_{f_{i j} \rightarrow x_{i, t}}^{(l)}\left(x_{i, t}\right)
$$

The location $x_{i, t}$ is estimated by the minimum mean-square error (MMSE) algorithm or maximum a posteriori (MAP) according to $b^{(\text {Niter })}\left(x_{i, t}\right)$. The detailed process of the BP algorithm of cooperative localization is presented in Algorithm 1.

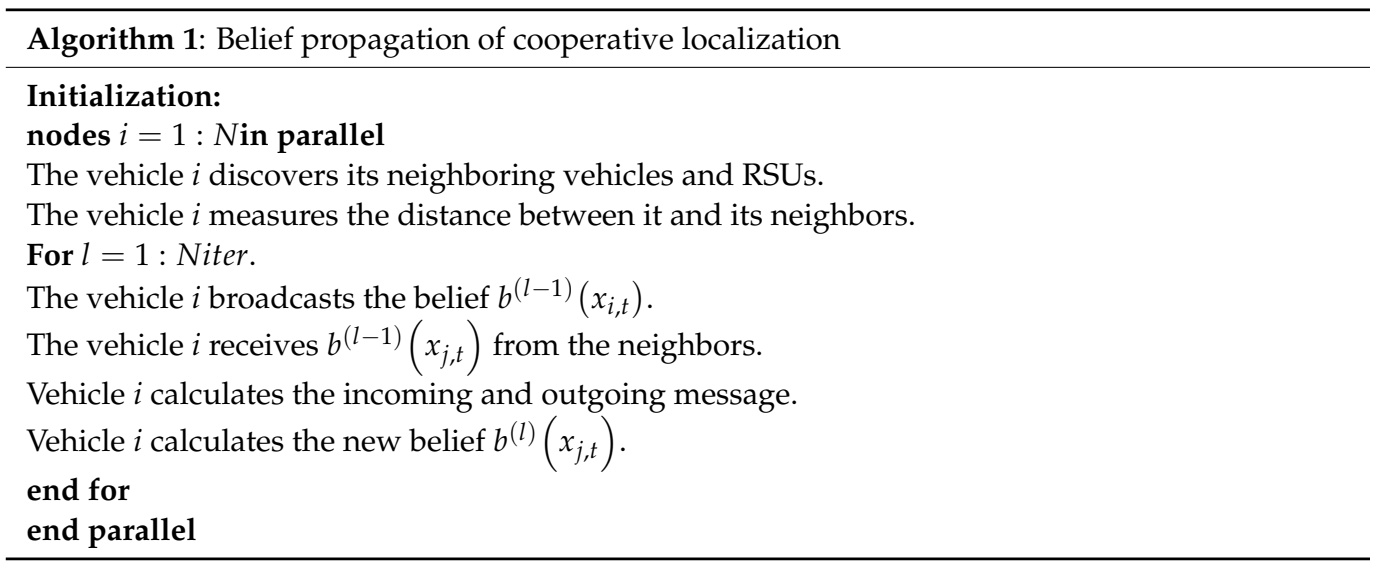

However, with the number of vehicles increasing, the BP algorithm has a high computational complexity and heavy communication overhead. For the vehicle networks, this may cause network congestion and even fail to locate.

\subsection{Evolutionary Coalitional Game Algorithm Based Cooperative Localizaion}

Since the evolutionary coalitional (EC) game is suitable for capturing wireless networks' behaviors in terms of randomness and uncertainty [21,22], we employ it to construct a stable coalition structure.

The EC game for the cooperative localization in vehicle networks is described as:

- Players: The finite vehicles join in the game of cooperative localization. The RSUs assist in vehicle localization but do not play a role in games.

- Population: All vehicles form coalitions, and each coalition belongs to a population.

- Strategy: The vehicle's strategy set is defined in the neighboring coalitions, and the vehicle determines its activities based on the actions of other vehicles.

- Payoff: The vehicle's payoff is influenced by its connected neighbor vehicles. Each vehicle determines which coalition to join according to the preference profile set during the formation and strategy learning stage.

The vehicle's payoff function $u(\cdot)$ is based on [23] and [18], which is composed of the benefit and cost of the vehicle. The $u(\cdot)$ is defined as

$$
u_{i, m}(t)=S P E B_{i, m}+\alpha \cdot \log \left(1-\left(\frac{\left|n_{i}^{(l)}\right|-\Delta}{\mathcal{N}_{i}}\right)^{2}\right)
$$

where $\alpha$ represents the balance parameter; $\mathcal{N}_{i}$ is the number of the $i$ vehicle's neighbors; $n_{i}$ denotes the number of nodes with the $i$ vehicle communication; and $\Delta$ is introduced to avoid $\left|n_{i}^{(l)}\right|=\mathcal{N}_{i}$. The benefit of this is the squared position error bound (SPEB), which is 
relevant to the information from anchors, the range information, and the prior knowledge of the vehicles' positions [23-25]. Moreover, the cost of the vehicle is the ratio of the number of communicating neighbors.

In the vehicle's utility, the number of neighboring vehicles needs to be considered. It is worth noting that more neighbor vehicles can improve the benefit of the node but that the cost is also increased. We denote $q_{i}(t)=\left[q_{i, m}\right]_{m \in S_{i}}$ as the preference profile set of the $i$ vehicle to select the coalition, where $S_{i}$ is the strategy set of the $i$ vehicle. The evolutionary game dynamics play a significant role in coalition games, which update the preference profile set for players. It is noted that the replicator dynamics is a simple and effective dynamic model that represents the probability of the player choosing to jump out of the current to another coalition. The definition is as follows:

$$
\rho_{i(m, n)}(t)=\frac{1}{n_{i}}\left[\bar{u}_{i, m}(t)-\bar{u}_{i, n}(t)\right]_{+}
$$

where + indicates that a value greater than 0 is selected. In addition to that, the average payoff of the $i$ vehicle is provided using the payoff function

$$
\bar{u}_{i, m}(t)=q_{i, m}(t)\left(\prod_{\substack{j \in \mathcal{F}_{m} \backslash\{i\} \\ j \in \mathcal{N}_{i}}} q_{j, m}(t)\right) u_{i, m}(t)
$$

where $q_{i, m}$ represents the probability of the $i$ vehicle selecting the $\mathcal{F}_{m}(t)$ coalition and $\mathcal{N}_{i}$ represents the neighboring vehicle set of the $i$ vehicle.

Additionally, the evolution of the coalition preference is related to the probability of change and given by:

$$
\begin{gathered}
\dot{q}_{i, m}(t)=\sum_{\mathcal{F}_{n} \in S_{i} \backslash \mathcal{F}_{m}} \rho_{i(n, m)}\left(x_{-i}\right) q_{i, n}(t)-q_{i, m}(t) \sum_{\mathcal{F}_{n} \in S_{i} \backslash \mathcal{F}_{m}} \rho_{i,(m, n)}\left(x_{-i}\right) \\
q_{i, m}(t+1)=q_{i, m}(t)+\dot{q}_{i, m}(t)
\end{gathered}
$$

If the evolution of coalition preference $\dot{q}_{i, m}$ is greater than 0 , the $i$ vehicle joins in the $m$ coalition. If $\dot{q}_{i, m}$ is equal to 0 , the $i$ vehicle keeps the current coalition. Otherwise, the $i$ vehicle departs the $m$ coalition.

The new strategic preferences based on continuous iterations are provided via the learning stage and a stable coalition structure. The evolutionary stable coalitional structure (ESCS) [26] is employed to represent the stable state of an EC game in which the vehicle coalitions are resilient to vehicle topology changes. Additionally, the perturbation of strategies represents the vehicles changing due to new vehicles joining, NLOS in a vehiclevehicle link, the message congestion of vehicles, and vehicles exiting the location. The ESCS is defined as

$$
\bar{u}_{i}\left(q_{i}^{\prime},(1-\epsilon) q_{-i}^{*}+\epsilon q_{-i}^{\prime}\right) \leq \bar{u}_{i}\left(q_{i}^{*},(1-\epsilon) q_{-i}^{*}+\epsilon q_{-i}^{\prime}\right),
$$

where $\epsilon \in(0,1)$ is a probability and $q_{-i}^{\prime}$ and $q_{i}^{*}$ are different strategy sets. Specifically, when $\epsilon$ vehicles in the coalition $q_{-i}^{*}$ change their strategy and quit the coalition $q_{-i}^{*}$ or $\epsilon$ portion of vehicles join in this coalition, the vehicles in the coalition $q_{i}^{*}$ still maintain the highest payoff.

A detailed illustration of cooperative localization with the EC game is revealed in Algorithm 2. We employ the EC game to select the neighboring vehicles to reduce the computational complexity and communication overhead. Vehicles form coalitions and exchange information with members of the coalition. While the number of iterations for cooperative localization is maintained, we manage to reduce the number of communication vehicles. 


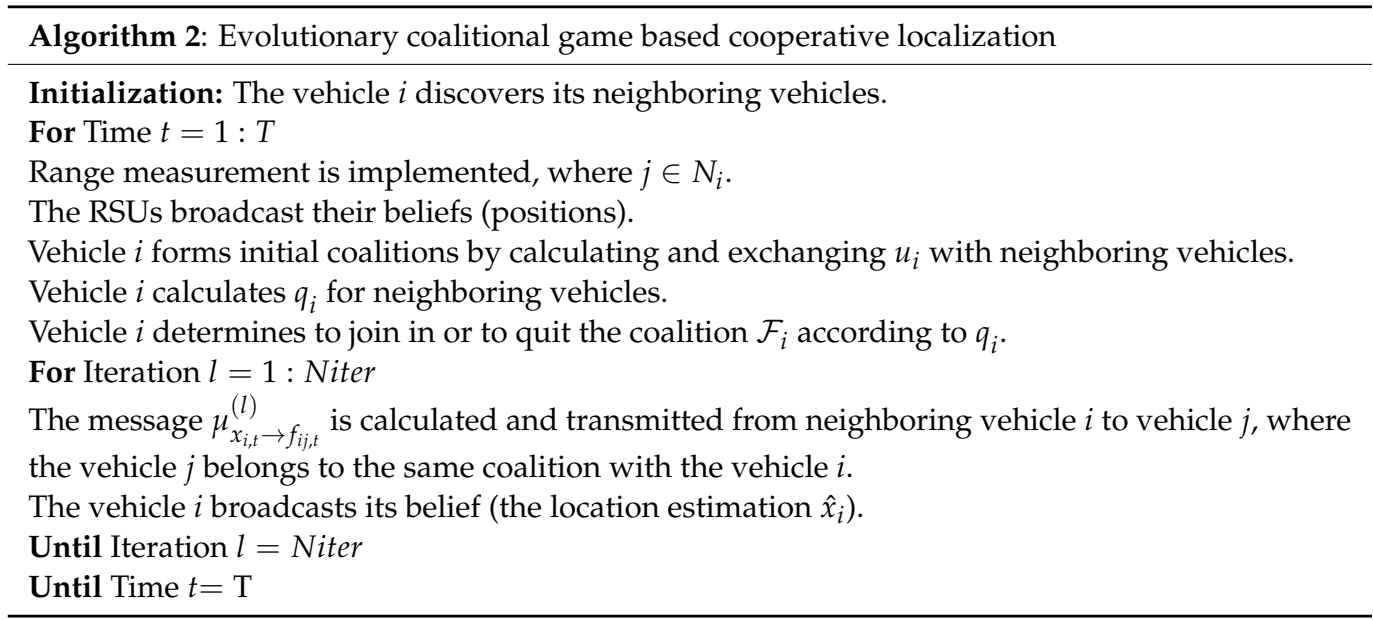

\section{Results}

The simulation deploys a $240 \mathrm{~m} \times 240 \mathrm{~m}$ crossroad with 20 fixed RSUs along the road and 80 randomly placed vehicles, where the distance between two RSUs is $40 \mathrm{~m}$. The considered scenario is described in Figure 2. The red triangle regions are the RSUs and the blue circles represent the vehicles' positions. The communication range is $30 \mathrm{~m}$. The vehicles carry out the same direction of movement with different speeds (with a range of 0-1 m/s) under the considered scenario. In addition to that, the distance between vehicles is set as greater than $6 \mathrm{~m}$ because the vehicle is usually $5 \mathrm{~m}$ long and $2 \mathrm{~m}$ wide. We assume that the coverage of RSUs is seamless and that each vehicle finds at least one RSU. The $\alpha$ is set to 1 , meaning that the two terms of payoff function can be well balanced.

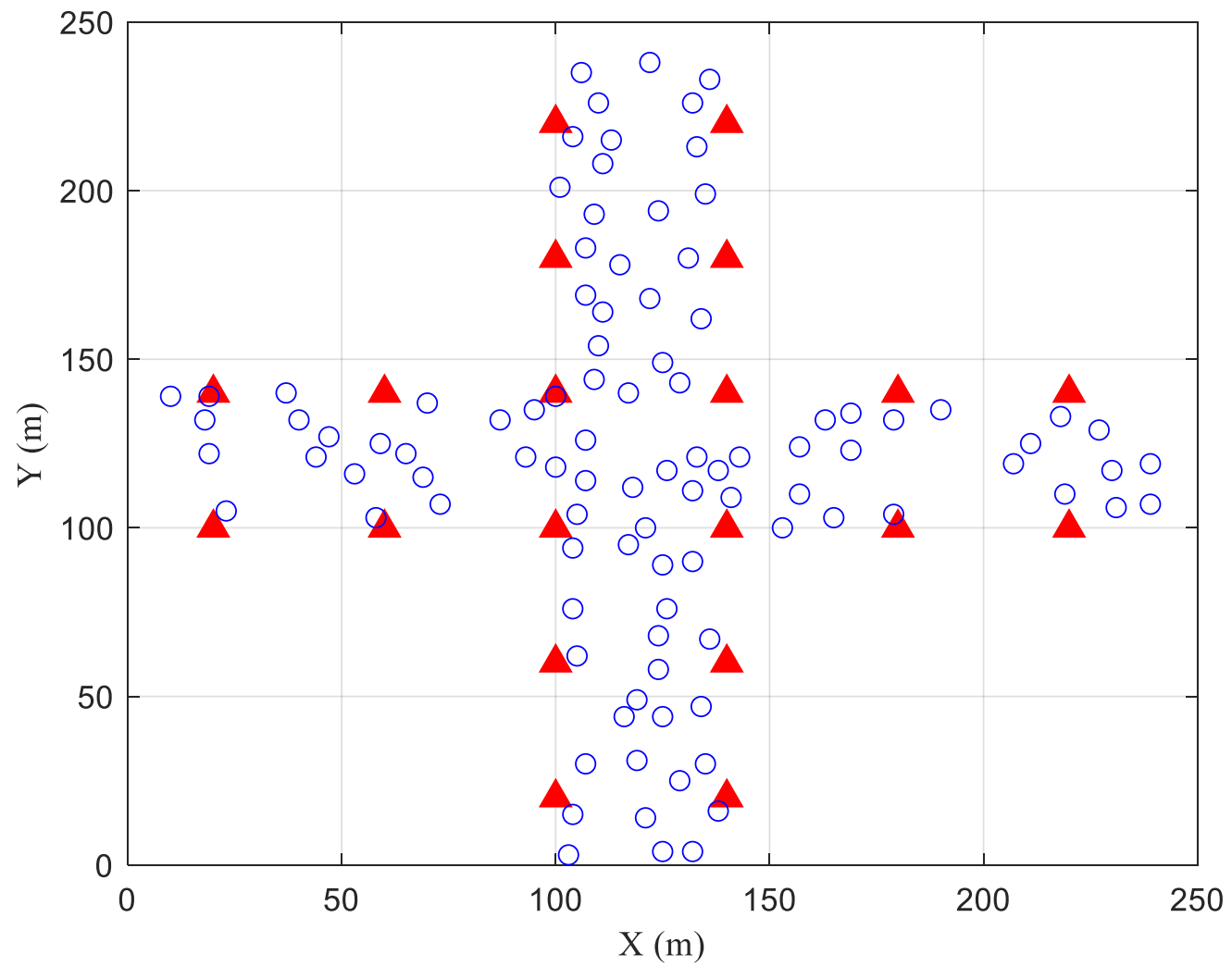

Figure 2. Scenario used for the simulations. The red triangle regions are the RSUs and the blue circles represent the vehicles' positions. 
Additionally, the RSU's position is drawn from a Gaussian posterior probability density function (PDF), whose mean is the true position and whose covariance is $0.1 \mathrm{~m}$. For the vehicle, its covariance is $10 \mathrm{~m}$. The measurement error $\mathrm{R}$ is $1 \mathrm{~m}$. The PLBP with a weight of $1 / 3$ and dimension of $4 \times 9$ sigma-points is employed to estimate the vehicles' positions. In the end, the $\mathrm{N}_{\text {iter }}$ is set to 5 .

Each vehicle has an a priori location with a low precision that obeys the Gaussian distribution. The vehicles learn the coalition strategy and form relatively stable coalitions for location estimation, utilizing the proposed method. The results are compared with the OCF game-based method and PLBP, which performs well for mobile networks as a typical cooperative localization algorithm.

\section{(1) Localization performance}

The RMSE of the proposed method over time in vehicle networks is shown in Figure 3. In the first few seconds, the positioning error of the proposed algorithm is larger than that of PLBP, as the EC game needs to achieve the ESCS. As the time taken increased, the proposed method's root means squared error (RMSE) achieved a value of $0.35 \mathrm{~m}$ at a speed of $0.1 \mathrm{~m} / \mathrm{s}$. This demonstrates that the RMSE in the proposed algorithm and the PLBP algorithm tends to coverage over time. According to the analysis method used in Ref. [18], the difference from the PLBP method can be neglected. However, for the OCF-based method, the localization accuracy is still poorer than that of other localization methods. Therefore, the localization performance degradation of the proposed method is minor.

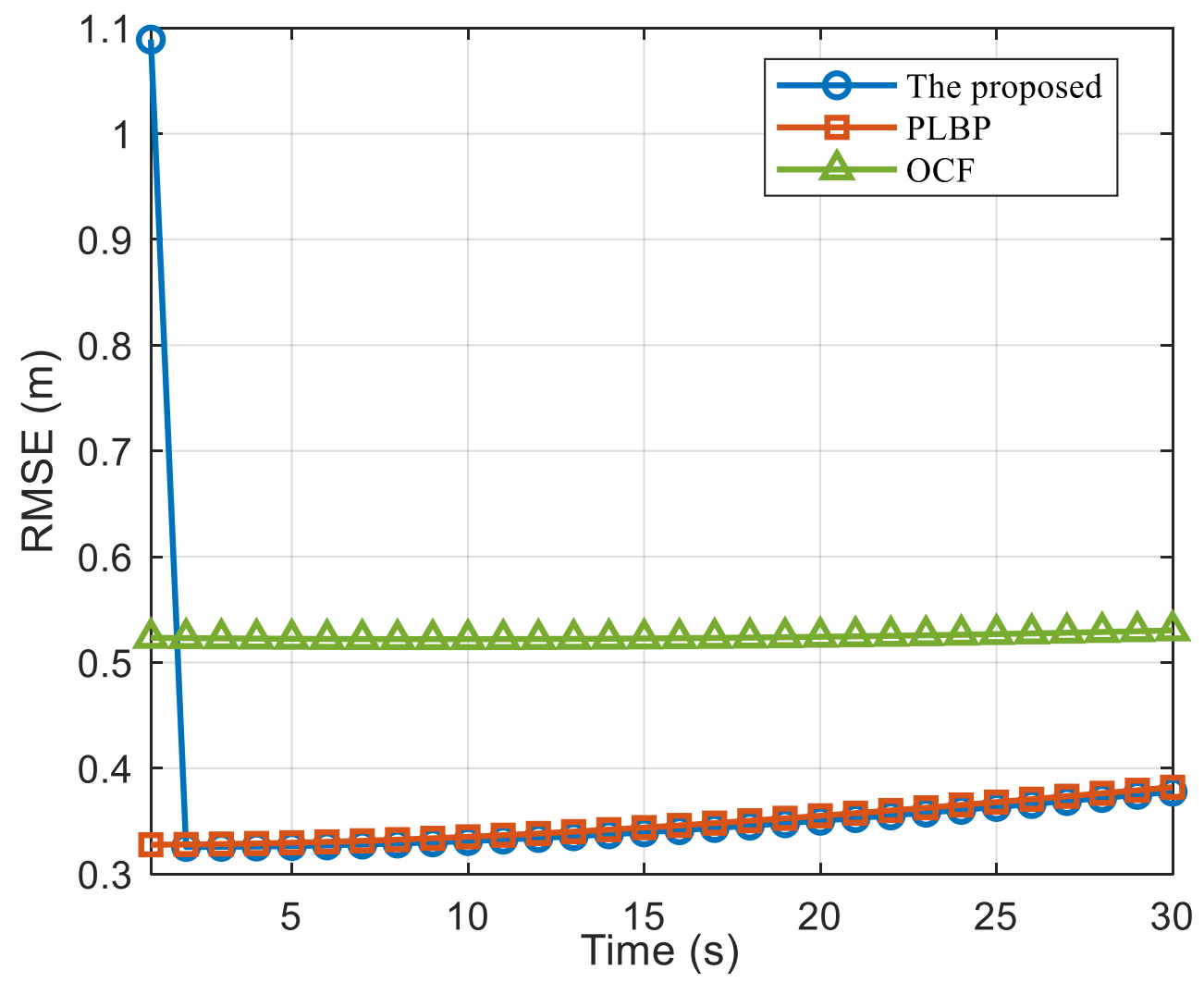

Figure 3. RMSE of the localization error.

Meanwhile, the vehicles with different speeds are described in Figure 4. The RMSEs of the proposed method are $0.39 \mathrm{~m}, 0.68 \mathrm{~m}$, and $0.99 \mathrm{~m}$ under the speeds of $0.1 \mathrm{~m} / \mathrm{s}, 0.5 \mathrm{~m} / \mathrm{s}$, and $1 \mathrm{~m} / \mathrm{s}$, respectively. Although the speed of the vehicle affects the localization error, this is because the localization algorithm does not utilize the velocity information of past moments, the proposed method has the same trend as the PLBP. Therefore, the proposed method does not affect the localization accuracy at the same speed. 


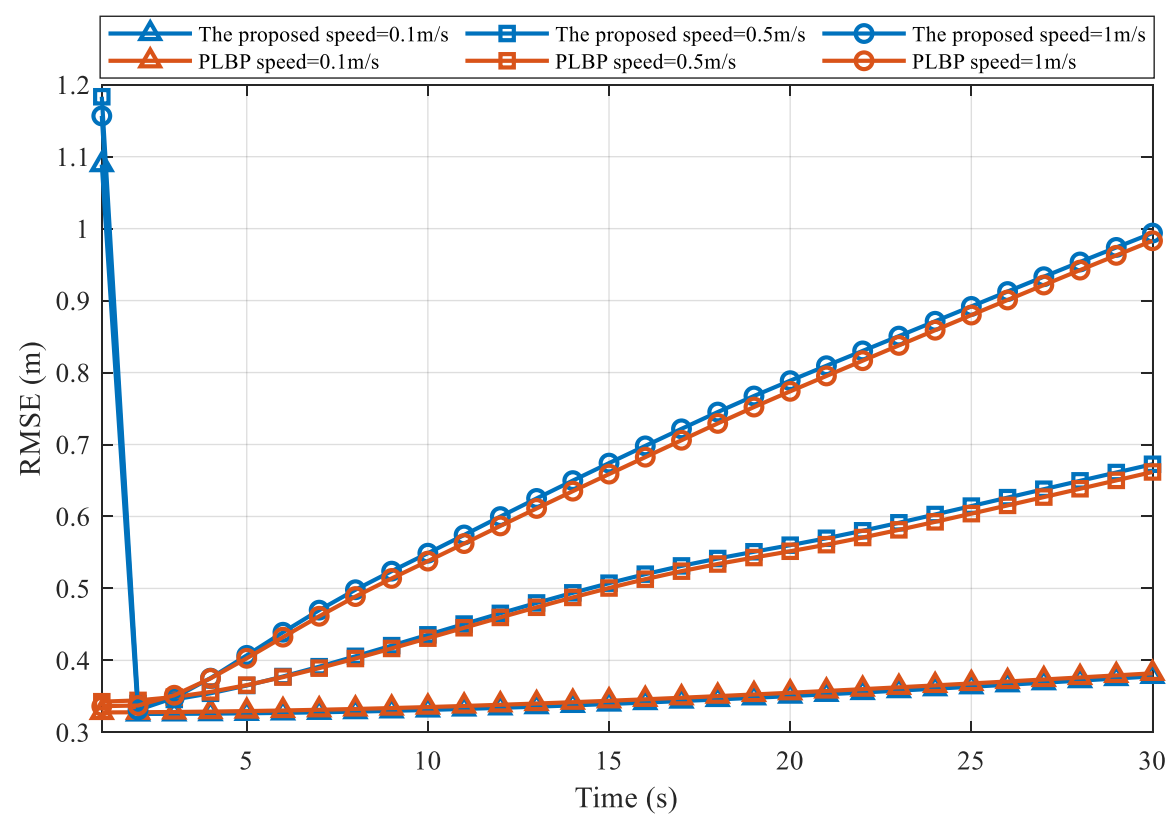

Figure 4. RMSE with different vehicle speeds for cooperative localization.

\section{(2) Complexity and Network Traffic}

The complexity of the cooperative localization algorithm is $\mathcal{O}\left(Q\left|n_{i}^{(l)}\right|\right)$, where $n_{i}$ denotes the average number of nodes positioned for the $i$ vehicles and $Q$ denotes the message representation complexity pre link. The complexity depends on the number of nodes communicating per vehicle. Figure 5 describes the comparison of $n_{i}$ for the proposed method, the OCF-based method, and PLBP with 5 iterations. The average number of nodes with the proposed method is 2 , which is less than the number in the OCF-based method ( 3 nodes on average) and the PLBP algorithm (11 nodes on average). The complexity of the proposed method is less than that of the conventional algorithm.

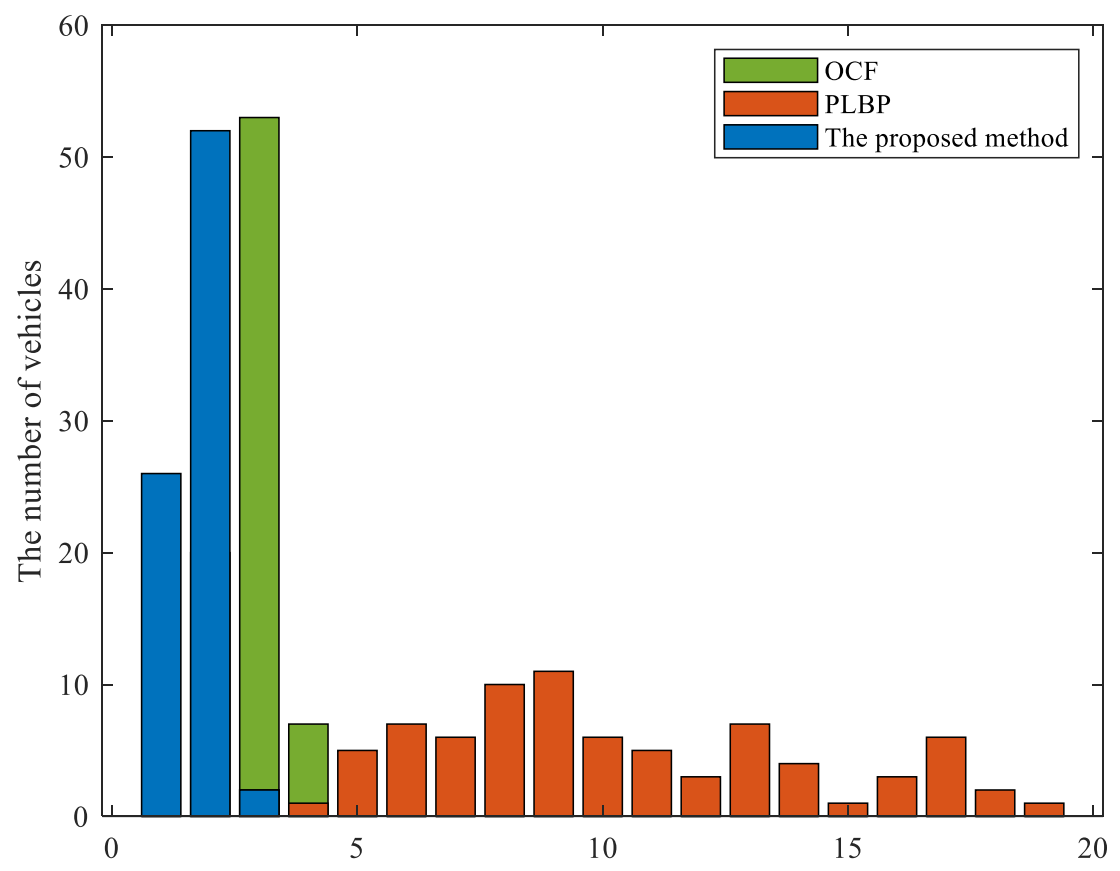

Average number of nodes for vehicle communication

Figure 5. Preference of potential coalition sizes for all vehicles. 


\section{Discussion}

Inaccurate positions, time synchronization, and erroneous measurements are the primary error sources in wireless localization [27]. Here, we discuss and analyze the influence of different error sources on localization performance. In the proposed localization method, we consider the SPEB and communication cost as the vehicle payoff in the EC game to form a localization coalition. The SPEB is composed of the RSU's location, range measurement information, and the a priori information of the vehicle. Moreover, communication cost is relevant to the number of neighbors in the coalition. Since the RSUs, as infrastructure, have precise location information, we consider the proposed localization method's error sources: time synchronization and measurement error. Therefore, the impact of above error sources is discussed.

For time synchronization, generally, users within the coverage area of a BS are timesynchronized with the BS in a cellular network. In V2V communication, vehicles within the coverage of the RSU are also synchronized with the RSU, while the vehicles in semicoverage or outside the coverage are synchronized with the vehicles that need to communicate [28]. However, since the requirements for time synchronization in positioning are much higher than those in communication, an effective time synchronization method is necessary. Similar to the BP algorithm, this proposed method is also affected by time synchronization [29]. The joint synchronization and localization algorithm can exchange the clock parameters of the vehicles while the positioning information is exchanged between the vehicles, thereby ensuring the time synchronization between the vehicles [30-32]. The above method can solve the problem of the impact of time synchronization on cooperative localization well, though we will not discuss it in detail here.

Moreover, the measurement error mainly affects the vehicle's SPEB. The SPEB of the vehicle is defined as:

$$
\mathrm{SPEB}_{k} \triangleq \operatorname{tr}\left\{\left[\mathbf{J}_{e}\left(\mathbf{P}_{k}\right)\right]_{2 \times 2, k}^{-1}\right\}
$$

Then, the $\mathbf{J}_{e}\left(\mathbf{P}_{k}\right)$ is given by:

$$
\mathbf{J}_{\mathbf{e}}\left(\mathbf{p}_{k}\right)=\sum_{j \in N_{a, b}} \lambda_{k j}\left(\left(1-\gamma^{2}\right) \beta^{2} \sum_{m=1}^{N_{t}} \mathbf{J}_{\mathbf{y}}\left(\phi_{k j}\right)+\frac{N_{t}\left(\gamma \beta+f_{c}\right)^{2} G_{k j}}{z_{k j}^{2}} \mathbf{J}_{\mathbf{y}}\left(\phi_{k j}+\frac{\pi}{2}\right)\right)
$$

From the formula, it can be seen that the distance $z_{k j}$ and angle $\phi_{k j}$ are variables that need to be measured. Therefore, it is affected by measurement errors.

The measurement error is set to $0 \mathrm{~m}, 1 \mathrm{~m}$, and $10 \mathrm{~m}$ with the vehicles having a $0.1 \mathrm{~m} / \mathrm{s}$ speed. Figure 6 shows the RMSE of the proposed method with the measurement error. We observe the influence of the measurement error on the localization accuracy of the vehicles. Note that the proposed localization method is barely affected by measurement errors, which is mainly due to the special network topology and the effective cooperative localization algorithm used. The roadside unit enables the vehicles to receive precise position information within a range of up to two hops, which greatly reduces the error accumulation. 


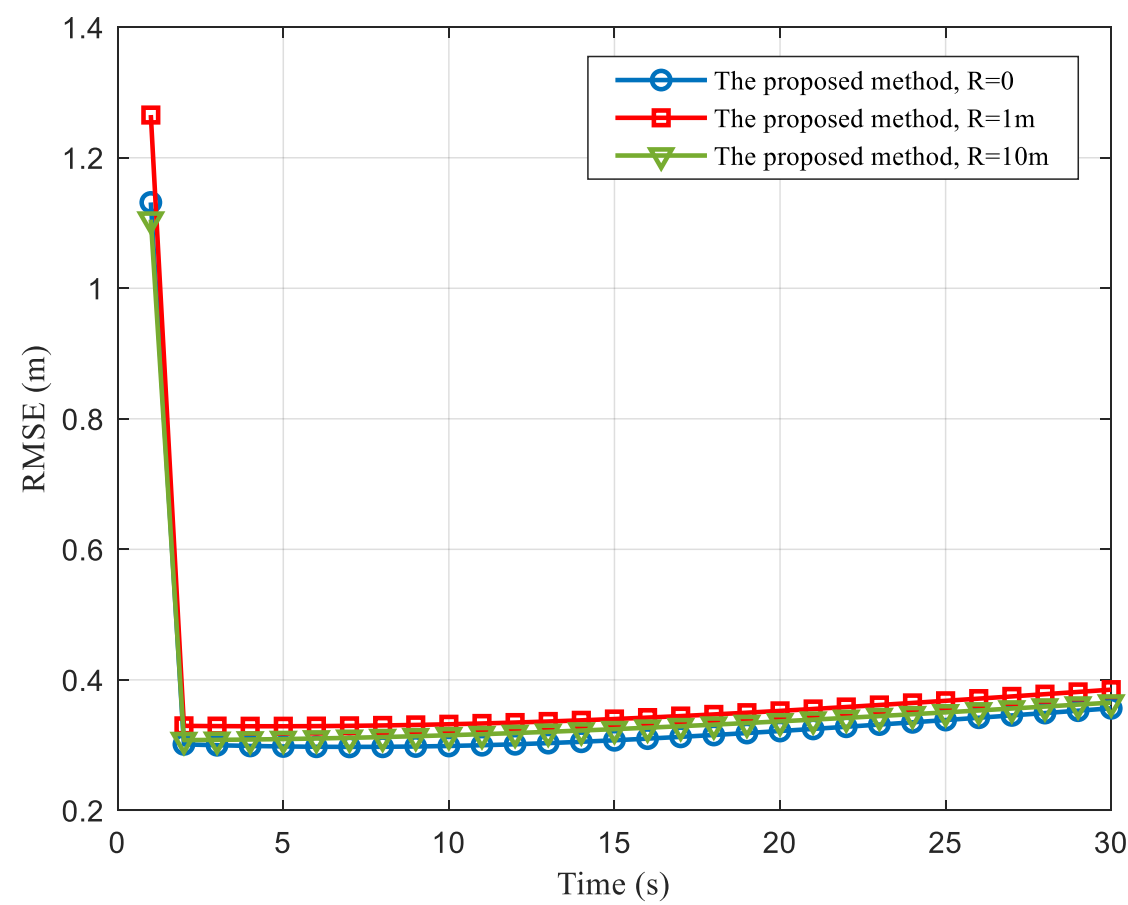

Figure 6. The RMSE with the measurement error.

\section{Conclusions}

In this paper, we focused on the limitations of cooperative localization in vehicle networks and proposed a fusion framework based on cooperative localization and the evolutionary coalitional game. Specifically, to reduce the high computational complexity and communication overhead, we conducted an evolutionary coalitional game to select the neighboring vehicles to form coalitions. In this method, the vehicle implements positioning via information exchange among neighboring nodes in the coalition. The simulation results indicate that the proposed evolutionary coalitional game-based cooperative localization model reduced the computational complexity and communication overhead while maintaining the localization accuracy. The proposed model results are thus beneficial for vehicle position identification.

In the future, we would like to apply the proposed framework in a practical cooperative localization system and address the specific problems brought about by network protocols.

Author Contributions: Conceptualization, T.Y. and D.Z.; methodology, T.Y.; software, T.Y.; validation, T.Y.; formal analysis, T.Y.; investigation, T.Y.; resources, T.Y.; data curation, T.Y.; writing-original draft preparation, T.Y.; writing-review and editing, C.B.; visualization, C.B.; supervision, X.L.; project administration, X.L.; funding acquisition, D.Z. All authors have read and agreed to the published version of the manuscript.

Funding: This research is supported by National Natural Science Foundation of China under Grant No: 41974032.

Institutional Review Board Statement: Not applicable.

Informed Consent Statement: Not applicable.

Data Availability Statement: The data used to support the findings of this study are available from the corresponding author upon request.

Acknowledgments: In this section, you can acknowledge any support given which is not covered by the author contribution or funding sections. This may include administrative and technical support, or donations in kind (e.g., materials used for experiments).

Conflicts of Interest: The authors declare no conflict of interest. 


\section{References}

1. Bounini, F.; Gingras, D.; Pollart, H.; Gruyer, D. From Simultaneous Localization and Mapping to Collaborative Localization for Intelligent Vehicles. IEEE Intell. Transp. Syst. Mag. 2021, 13, 196-216. [CrossRef]

2. Cao, Z.; Yang, D.; Jiang, K.; Xu, S. Monocular Connected-Vehicle Position Estimation on Sloping and Uneven Roads. IEEE Intell. Transp. Syst. Mag. 2022, 14, 228-241. [CrossRef]

3. Liu, D.; Cui, Y.; Guo, X.; Ding, W.; Yang, B.; Chen, Y. Visual Localization for Autonomous Driving: Mapping the Accurate Location in the City Maze. In Proceedings of the 2020 25th International Conference on Pattern Recognition (ICPR), Milan, Italy, 10-15 January 2021; pp. 3170-3177.

4. Yuan, W.; Liu, F.; Masouros, C.; Yuan, J.; Ng, D.W.K.; Gonzalez-Prelcic, N. Bayesian Predictive Beamforming for Vehicular Networks: A Low-Overhead Joint Radar-Communication Approach. IEEE Trans. Wireless Commun. 2021, 20, 1442-1456. [CrossRef]

5. Kassas, Z.Z.M.; Maaref, M.; Morales, J.J.; Khalife, J.J.; Shamei, K. Robust Vehicular Localization and Map Matching in Urban Environments Through IMU, GNSS, and Cellular Signals. IEEE Intell. Transp. Sys. Mag. 2020, 12, 36-52. [CrossRef]

6. Eskandarian, A.; Wu, C.; Sun, C. Research Advances and Challenges of Autonomous and Connected Ground Vehicles. IEEE Trans. Intell. Transp. Sys. 2021, 22, 683-711. [CrossRef]

7. Wymeersch, H.; Seco-Granados, G.; Destino, G.; Dardari, D.; Tufvesson, F. 5G mmWave Positioning for Vehicular Networks. IEEE Wirel. Commun. 2017, 24, 80-86. [CrossRef]

8. 3GPP TS38.305; NG Radio Access Network (NG-RAN); User Equipment (UE) Positioning in NG-RAN. 3GPP: Sophia Antipolis, France, 2019.

9. Kim, H.; Granstrom, K.; Gao, L.; Battistelli, G.; Kim, S.; Wymeersch, H. 5G mmWave Cooperative Positioning and Mapping Using Multi-Model PHD Filter and Map Fusion. IEEE Trans. Wireless Commun. 2020, 19, 3782-3795. [CrossRef]

10. Luo, F.; Wang, S.; Gong, Y.; Jing, X.; Zhang, L. Geographical Information Enhanced Cooperative Localization in Vehicular Ad-Hoc Networks. IEEE Signal Process. Lett. 2018, 25, 556-560. [CrossRef]

11. Meng, W.; Chu, X.; Lu, Z.; Wang, L.; Wen, X.; Li, M. V2V Communication Assisted Cooperative Localization for Connected Vehicles. In Proceedings of the 2021 IEEE Wireless Communications and Networking Conference (WCNC), Nanjing, China, 29 March-1 April 2021; pp. 1-6.

12. Wymeersch, H.; Lien, J.; Win, M.Z. Cooperative Localization in Wireless Networks. Proc. IEEE 2009, 97, 427-450. [CrossRef]

13. Ihler, A.; Fisher, J.; Moses, R.; Willsky, A. Nonparametric belief propagation for self-localization of sensor networks. IEEE J. Sel. Areas Commun. 2005, 23, 809-819. [CrossRef]

14. García-Fernández, Á.F.; Svensson, L.; Särkkä, S. Cooperative Localization Using Posterior Linearization Belief Propagation. IEEE Trans. Veh. Technol. 2018, 67, 832-836. [CrossRef]

15. Tang, C.; Dou, L. An Improved Game Theory-Based Cooperative Localization Algorithm for Eliminating the Conflicting Information of Multi-Sensors. Sensors 2020, 20, 5579. [CrossRef] [PubMed]

16. Zhao, Z.; Zhang, R.; Cheng, X.; Yang, L.; Jiao, B. Network formation games for the link selection of cooperative localization in wireless networks. In Proceedings of the 2014 IEEE International Conference on Communications (ICC), Sydney, NSW, Australia, 10-14 June 2014; pp. 4577-4582.

17. Hedhly, K.; Laaraiedh, M.; AbdelKefi, F.; Siala, M. Optimizing communication costs based on novel cooperative localization techniques using coalitional game theory in wireless networks. In Proceedings of the 2018 14th International Wireless Communications \& Mobile Computing Conference (IWCMC), Limassol, Cyprus, 25-29 June 2018; pp. 1043-1046.

18. Zhang, R.; Zhao, Z.; Cheng, X.; Yang, L. Overlapping Coalition Formation Game Based Opportunistic Cooperative Localization Scheme for Wireless Networks. IEEE Trans. Commun. 2017, 65, 3629-3642.

19. Tian, K.M.; Tong, S.; Zhang, K. An EKF based overlapping coalition formation game for cooperative wireless network navigation. IET Commun. 2021, 15, 2407-2424.

20. Abu-Shaban, Z.; Wymeersch, H.; Abhayapala, T.; Seco-Granados, G. Distributed Two-Way Localization Bounds for 5G mmWave Systems. In Proceedings of the 2018 IEEE Globecom Workshops (GC Wkshps), Abu Dhabi, United Arab Emirates, 9-13 December 2018; pp. 1-6.

21. Khan, M.A.; Tembine, H.; Vasilakos, A.V. Evolutionary coalitional games: Design and challenges in wireless networks. IEEE Wireless Commun. 2012, 19, 50-56. [CrossRef]

22. Asheralieva, A.; Quek, T.Q.S.; Niyato, D. An Asymmetric Evolutionary Bayesian Coalition Formation Game for Distributed Resource Sharing in a Multi-Cell Device-to-Device Enabled Cellular Network. IEEE Trans. Wireless Commun. 2018, 17, $3752-3767$. [CrossRef]

23. Shen, Y.; Wymeersch, H.; Win, M.Z. Fundamental Limits of Wideband Localization—Part II: Cooperative Networks. IEEE Trans. Inform. Theory 2010, 56, 4981-5000. [CrossRef]

24. Mazuelas, S.; Shen, Y.; Win, M.Z. Spatiotemporal information coupling in network navigation. IEEE Trans. Inform. Theory 2018, 64, 7759-7779. [CrossRef]

25. Xiong, Y.; Wu, N.; Shen, Y.; Win, M.Z. Cooperative localization in massive networks. IEEE Trans. Inform. Theory 2021, 68, 1-23. [CrossRef]

26. Luo, X.; Tembine, H. Evolutionary coalitional games for random access control. In Proceedings of the 2013 Proceedings IEEE INFOCOM, Turin, Italy, 14-19 April 2013; pp. 535-539. 
27. Yuan, W.; Wu, N.; Guo, Q.; Huang, X.; Li, Y.; Hanzo, L. TOA-Based Passive Localization Constructed Over Factor Graphs: A Unified Framework. IEEE Trans. Commun. 2019, 67, 6952-6965. [CrossRef]

28. 3GPP TS 36.331; Group Radio Access Network; Evolved Universal Terrestrial Radio Access (E-UTRA) and Evolved Universal Terrestrial Radio Access Network (E-UTRAN). 3GPP: Sophia Antipolis, France, 2017.

29. Xiong, Y.; Wu, N.; Shen, Y.; Win, M.Z. Cooperative Network Synchronization: Asymptotic Analysis. IEEE Trans. Signal Process. 2018, 66, 757-772. [CrossRef]

30. Yuan, W.; Wu, N.; Etzlinger, B.; Wang, H.; Kuang, J. Cooperative Joint Localization and Clock Synchronization Based on Gaussian Message Passing in Asynchronous Wireless Networks. IEEE Trans. Veh. Technol. 2016, 65, 7258-7273. [CrossRef]

31. Etzlinger, B.; Meyer, F.; Hlawatsch, F.; Springer, A.; Wymeersch, H. Cooperative Simultaneous Localization and Synchronization in Mobile Agent Networks. IEEE Trans. Signal Process. 2017, 65, 3587-3602. [CrossRef]

32. Yuan, W.; Yuan, J.; Kwan Ng, D.W. Parametric Message-Passing for Joint Localization and Synchronization in Cooperative Networks. In Proceedings of the 2020 IEEE Global Communications Conference (GLOBECOM), Taipei, Taiwan, 7-11 December 2020; pp. 1-6. 